\title{
O FINANCIAMENTO DAS UNIVERSIDADES ESTADUAIS BRASILEIRAS: O CUSTO ALUNO, AS ASSIMETRIAS INSTITUCIONAIS E AS DESIGUALDADES REGIONAIS
}

\author{
THE BRAZILIAN STATE UNIVERSITIES FUNDING: \\ THE SPENDING PER-STUDENT, THE INSTITUTIONAL ASYMMETRIES, AND THE \\ REGIONAL INEQUALITIES
}

\author{
Renata Ramos da Silva Carvalho \\ Doutorado em Educação (UFG) \\ Universidade Estadual de Goiás, Unidade Universitária de Inhumas \\ UEG UnU Inhumas. \\ Inhumas, Goiás - Brasil. \\ renataramosprof@yahoo.com.br \\ iD Nelson Cardoso Amaral \\ Doutor em Educação (UNIMEP). \\ Universidade Federal de Goiás - UFG. \\ Goiânia, Goiás - Brasil. \\ nelsoncardosoamaral@gmail.com
}

\begin{abstract}
Resumo: $\mathrm{O}$ artigo apresenta resultado de pesquisa que apurou o valor do custo aluno das universidades estaduais (UEs) brasileiras, por estado da federação, no período de 2012 a 2016, tendo como referência metodológica a abordagem proposta por Amaral e Pinto (2010), informações estatísticas das UEs presentes nos dados (sinopses estatísticas e microdados) do Censo da Educação Superior do Instituto Nacional de Pesquisas Educacionais Anísio Teixeira (Inep) e da Coordenação de Aperfeiçoamento de Nível Superior (Capes). Os resultados obtidos mostram que os valores do custo aluno das UEs são assimétricos e revelam desigualdades regionais existentes quanto a atuação dessas instituições no contexto da educação superior do país.
\end{abstract}

Palavras-chave: assimetrias regionais; custo aluno; financiamento; universidades estaduais.

Abstract: The article presents a research result that verified the average spending per-student of the Brazilian State Universities (UEs), by state of the federation, in the period between 2012 and 2016. As a methodological reference, the article presents the approach proposed by Amaral and Pinto (2010), statistical information of the UEs present in the data (statistical abstracts and microdata) of the National Institute for Educational Studies and Research "Anísio Teixeira" (Inep), and of the Coordination for the Improvement of Higher Education Personnel (Capes). The results obtained show that the average spending per-student of the UEs are asymmetrical and reveal existing regional inequalities regarding the performance of these institutions in the higher education context of the country.

Palavras-chave: regional asymmetries; spending per-student; funding; state universities.

\section{Para citar - (ABNT NBR 6023:2018)}

CARVALHO, Renata Ramos da Silva; AMARAL, Nelson Cardoso. O financiamento das universidades estaduais brasileiras: o custo aluno, as assimetrias institucionais e as desigualdades regionais. Eccos - Revista Científica, São Paulo, n. 57, p. 1-20, e10777, abr./jun. 2021. Disponível em: https://doi.org/10.5585/eccos.n57.10777. 


\section{Introdução}

A promulgação da CF de 1988 constituiu-se como um importante marco na definição dos princípios e finalidades que sustentam a educação nacional. Nela, a educação foi definida como um dos direitos sociais do cidadão brasileiro. Desse modo, o texto constitucional expressa que a educação é "direito de todos e dever do Estado e da família” (BRASIL, 1988, art. 205) e ainda define que ela será ministrada baseada pelos princípios, dentre outros, da igualdade de condições para o acesso e permanência na escola, gratuidade do ensino público em estabelecimentos oficiais e garantia de padrão de qualidade.

A organização da educação brasileira, disposta na CF de 1988, estabelece atribuições que competem à União, aos estados, aos municípios e ao Distrito Federal. Quanto à atuação e competências dos entes federados, ela disciplina que eles organizarão os seus sistemas de ensino em regime de colaboração e ainda define que:

\footnotetext{
$\S 1^{\circ}$ A União organizará o sistema federal de ensino e o dos Territórios, financiará as instituições de ensino públicas federais e exercerá, em matéria educacional, função redistributiva e supletiva, de forma a garantir equalização de oportunidades educacionais e padrão mínimo de qualidade do ensino mediante assistência técnica e financeira aos Estados, ao Distrito Federal e aos Municípios;

$\S 2^{\circ}$ Os Municípios atuarão prioritariamente no ensino fundamental e na educação infantil.

$\S 3^{\circ}$ Os Estados e o Distrito Federal atuarão prioritariamente no ensino fundamental e médio.

$\S 4^{\circ} \mathrm{Na}$ organização de seus sistemas de ensino, a União, os Estados, o Distrito Federal e os Municípios definirão formas de colaboração, de modo a assegurar a universalização do ensino obrigatório.

$\S 5^{\circ}$ A educação básica pública atenderá prioritariamente ao ensino regular. (BRASIL, 1988, art. 211. Grifos da autora)
}

Quanto à definição de atribuições na oferta dos níveis educacionais estabelecidos pelos supracitados parágrafos, infere-se que os governos municipais, estaduais e o distrital devem responsabilizar-se, prioritariamente, pela educação básica, cabendo à União a função redistributiva e supletiva que possibilite a equalização das oportunidades educacionais mediante assistência técnica e financeira aos demais entes federados. A CF de 1988 não define a competência acerca da oferta de educação superior pública, não havendo expresso, no ordenamento jurídico brasileiro, a previsão de qual ente federado será o responsável, mesmo que prioritariamente, pela sua oferta pública. No que diz respeito ao financiamento destinado à educação, as disposições contidas no artigo 212 da CF de 1988 preveem os percentuais mínimos da arrecadação de impostos de cada ente federado para serem aplicadas na educação. Está expresso que "a União aplicará, anualmente, nunca menos de dezoito, e os Estados, o Distrito 
Federal e os Municípios vinte e cinco por cento, no mínimo, da receita resultante de impostos.” (Brasil, 1988).

Considerando o regime de competências e as atribuições previstas aos estados da federação pela LDB/1996, em seu artigo 10, embora a competência específica desses entes federados seja a de assegurar a oferta da educação básica, em especial o ensino médio, eles podem ofertar educação superior pública estadual. Essa prerrogativa permite aos estados brasileiros ofertarem educação superior pública, cabendo-lhes a responsabilidade pelo seu financiamento e os processos que envolvem autorização, reconhecimento, credenciamento, supervisão e avaliação das IES que lhes são subordinadas, com exceção da oferta de cursos de educação a distância ${ }^{1}$.

Os estudos de Carvalho (2013), Fialho (2012, 2011, 2005), Sampaio, Balbachesky e Peñaloza (1998), teceram considerações acerca da relevância das UEs na oferta de educação superior pública nas localidades em que estão instaladas, em especial, quanto a relevância delas no processo de interiorização deste nível educacional. Essas autoras também discutiram aspectos relacionados aos desafios quanto as condições materiais e acadêmicas das UEs para promoverem suas atividades de ensino, pesquisa e extensão. No ano de 2016, segundo dados do Censo da Educação Superior do Inep, existiam $39^{2}$ UEs no país. Conforme detalhamento do quadro 1, elas foram responsáveis por $27,5 \%$ das matrículas públicas no país e por 32,6\% das matrículas em universidades públicas no Brasil.

Quadro 1 - relação e distribuição das UEs brasileiras, por região e estado da federação, ano de criação e quantidade de campus/unidades universitárias

\begin{tabular}{|c|l|}
\hline Estado & \multicolumn{1}{c|}{ Instituições } \\
\hline & Norte \\
\hline RR & Universidade Estadual de Roraima - UERR \\
\hline TO & Fundação Universidade do Tocantins - Unitins \\
\hline AP & Universidade do Estado do Amapá - Ueap \\
\hline AM & Universidade do Estado do Amazonas - UEA \\
\hline PA & Universidade do Estado do Pará - Uepa \\
\hline & Nordeste \\
\hline PB & Universidade Estadual da Paraíba - UEPB \\
\hline BA & $\begin{array}{l}\text { Universidade Estadual de Feira de Santana - UEFS } \\
\text { Universidade Estadual do Sudeste da Bahia - Uesb } \\
\text { Universidade Estadual de Santa Cruz - Uesc } \\
\text { Universidade do Estado da Bahia - Uneb }\end{array}$ \\
\hline PI & Universidade Estadual do Piauí - Uespi \\
\hline
\end{tabular}

\footnotetext{
${ }^{1}$ O Decreto Federal n. ${ }^{\circ} 9.057$, de 25 de maio de 2017, estabelece, no art. $6^{\circ}$, que "Compete ao Ministério da Educação em articulação com os órgãos e as entidades a eles vinculados: I - o credenciamento e o recredenciamento de instituições de ensino federal, estaduais e distrital para a oferta de educação superior na modalidade a distância." (BRASIL, 2017, p. 2).

${ }^{2}$ Para este estudo, será considerado 38 UEs, pois a Universidade Virtual de São Paulo (Univesp) só passou a constar no Censo no ano de 2016, com isso não possui série histórica de dados institucionais.
} 


\begin{tabular}{|c|l|}
\hline \multicolumn{1}{|c}{} & \multicolumn{1}{l}{ Conclusão } \\
\hline MA & Universidade Estadual do Maranhão - Uema \\
\hline PE & Universidade de Pernambuco - UPE \\
\hline AL & $\begin{array}{l}\text { Universidade Estadual do Ceará - Uece } \\
\text { Universidade Estadual do Cariri - Urca } \\
\text { Universidade Estadual Vale do Acaraú - UVA }\end{array}$ \\
\hline RN & $\begin{array}{l}\text { Universidade Estadual de Alagoas - Uneal } \\
\text { Universidade de Ciências da Saúde de Alagoas - Uncisal }\end{array}$ \\
\hline RJ & $\begin{array}{l}\text { Universidade do Estado do Rio Grande do Norte - Uern } \\
\text { Universidade Esto Estado do Rio de Janeiro - Uerj }\end{array}$ \\
\hline MG & $\begin{array}{l}\text { Universidade do Estado de Minas Gerais - UEMG } \\
\text { Universidade Estadual de Montes Claros - Unimontes }\end{array}$ \\
\hline SP & $\begin{array}{l}\text { Universidade de São Paulo - USP } \\
\text { Universidade Est. Paulista Júlio de Mesquita Filho - Unesp } \\
\text { Universidade Estadual de Campinas - Unicamp } \\
\text { Universidade Virtual de São Paulo - Univesp }\end{array}$ \\
\hline PR & $\begin{array}{l}\text { Universidade Estadual de Londrina - UEL } \\
\text { Universidade Estadual de Maringá - UEM } \\
\text { Universidade Estadual do Norte do Paraná - Uenp } \\
\text { Universidade Estadual de Ponta Grossa - UEPG } \\
\text { Universidade Est. do Centro Oeste do Paraná - Unicentro } \\
\text { Universidade Estadual do Oeste do Paraná - Unioeste } \\
\text { Universidade Estadual do Paraná - Unespar }\end{array}$ \\
\hline SC & Universidade do Estado de Santa Catarina - Udesc \\
\hline RS & Universidade Estadual do Rio Grande do Sul - Uergs \\
\hline MS & Universidade Estadual de Mato Grosso do Sul - Uems \\
\hline MT & Universidade Estadual de Mato Grosso - Unemat \\
\hline GO & Universidade Estadual de Goiás - UEG \\
\hline
\end{tabular}

Fonte: Inep - Censo da Educação Superior - 2016. Sítios eletrônicos das UEs. Sistematização realizada por este estudo.

Assim, este estudo apresentará os valores referentes ao custo aluno das UEs do país, por estado da federação, no período de 2012 a 2016, tendo como referência a metodologia proposta por Amaral e Pinto (2010), e informações estatísticas coletadas no banco de dados do Inep e da Capes.

\section{O financiamento das UEs: o custo médio do aluno, no período de 2012 a 2016}

Amaral e Pinto (2010, p. 61) ressaltam que um dos aspectos que é ponto de debate relacionado ao financiamento das IES, "é o quanto se gasta para formar um estudante de graduação, o que é comumente chamado de custo aluno”. Os autores esclarecem que há muitos equívocos nessa análise, "quando simplesmente se divide o gasto total da instituição pelo número de estudantes de graduação" (Ibid., p. 61). Nesse caso, o valor obtido seria o gasto institucional por aluno e não o custo do aluno, uma vez que há despesas, entre outras, com pesquisa e extensão que não podem ser consideradas para esse cálculo.

Nessa direção, Amaral e Pinto (2010, p. 55) desenvolveram uma metodologia de cálculo que possibilita apurar o custo médio do aluno de graduação na educação superior por instituição 
(ou instituições), que leva em consideração as seguintes características de uma IES: "percentual de professores em tempo integral; percentual de mestres e doutores; percentual de estudantes de pós-graduação; e a média dos conceitos Capes dos cursos de pós-graduação". Os autores esclarecem que,

Para realizarmos comparações consistentes entre as instituições, é preciso, entretanto, efetivar modelagens que permitam apurar o custo médio do aluno de graduação. Não há sentido na comparação entre o custo de um estudante de uma IES que desenvolve plenamente as atividades de ensino de graduação e pós-graduação, pesquisa, interação forte com a sociedade, mantém museus, orquestras, hospitais etc., com o custo do estudante de outra IES que só oferece cursos de graduação e desenvolve poucas atividades de pesquisa e de interação com a sociedade. (AMARAL; PINTO, 2010, p. 61)

Sendo assim, é indispensável considerar a dessemelhança existente entre as IES no país para a elaboração de uma metodologia que vislumbre apurar o custo médio do aluno de graduação, que é o caso das UEs brasileiras. Assim como no conjunto das IES brasileiras, a heterogeneidade das UEs também

se reflete, principalmente, nas atividades de pesquisa e extensão das instituições, e dependem fundamentalmente do número de mestres e doutores em cada uma delas. Existem, no Brasil, instituições que, no estágio em que se encontram, dedicam-se mais à pesquisa e à extensão; outras que se dedicam mais ao ensino e muitas que se dedicam exclusivamente ao ensino de graduação. Existem instituições com corpo docente altamente titulado e outras que se encontram em um estágio anterior, ainda promovendo a qualificação de seus professores. (AMARAL; PINTO, 2010, p. 62)

Tendo em vista esse cenário de assimetria existente no contexto da educação superior brasileira, bem como a necessidade de elaboração de uma metodologia para auferir o custo do aluno em IES no Brasil, Amaral e Pinto (2010) desenvolveram uma modelagem de cálculo para esse fim, que considera os seguintes aspectos:

1) Existe uma relação direta entre as despesas com pesquisa ou extensão e a quantidade de professores que trabalham em tempo integral nas instituições. Consideraremos nesta modelagem que os percentuais apurados nos itens 2, 3 e 4, a seguir, serão multiplicados por um fator que os relativize em relação ao percentual de professores em tempo integral (Fator Tempo Integral - FTI). O FTI será igual à fração de professores em tempo integral em relação ao total de professores das instituições. Então, se, por exemplo, $72 \%$ dos professores estão em tempo integral, o FTI associado será de 0,72 .

2) Existe uma relação direta entre o percentual das despesas que se destinam à pesquisa ou à extensão e o número de mestres e doutores.

A categoria administrativa (federal, estadual, municipal, particular, com/conf/ fil) que tiver $100 \%$ de seus professores com titulação de mestre ou doutor dedicará $50 \%$ de suas despesas para pesquisa ou extensão e aplicará $0 \%$ em pesquisa ou extensão se tiver nenhum de seus professores com essas titulações, relativizados pelo fator FTI. Valores entre 0\% e 100\% de professores mestres ou doutores significarão, proporcionalmente, gastos com pesquisa ou extensão entre $0 \%$ e $50 \%$, relativizados pelo FTI.

3) Existe uma relação direta entre as despesas com pesquisa ou extensão e o percentual de alunos de pós-graduação stricto sensu em relação ao total de alunos 
das instituições - graduação presencial adicionados aos de pós-graduação stricto sensu.

A modelagem deste estudo considera que o percentual de alunos de pós-graduação stricto sensu e o total de alunos será o percentual dedicado às atividades de pesquisa ou extensão relativizados pelo FTI que deve ser adicionado ao percentual do item anterior.

4) Existe uma relação direta entre as despesas com pesquisa ou extensão e a avaliação Capes de seus programas de pós-graduação stricto sensu.

Essa modelagem considera que, se a média do conceito Capes dos programas de pós-graduação for igual a sete, $10 \%$ dos recursos das instituições se dirigem à pesquisa ou à extensão relativizados pelo FTI; se a média for menor ou igual a três, $3 \%$ dos recursos se dirigem à pesquisa ou à extensão relativizados pelo FTI. Valores médios dos conceitos Capes entre três e sete significarão, proporcionalmente, gastos com pesquisa ou extensão entre $3 \%$ e $10 \%$ relativizados pelo FTI. O percentual apurado neste item deve ser adicionado aos percentuais dos itens $1 \mathrm{e} 2$.

Nessa modelagem, o maior valor para os gastos com pesquisa ou extensão ficaria com aquele conjunto de instituições - federal, estadual, municipal, particular, com/conf/fil - que satisfizesse, simultaneamente, as seguintes condições: 1) possuísse o maior percentual de professores em tempo integral, 2) todos os docentes fossem mestres ou doutores, 3) possuísse o maior número relativo de alunos de mestrado e doutorado entre os alunos das instituições e 4) possuísse o maior valor para a média dos conceitos Capes.[...]

Portanto, para o modelo do cálculo do custo médio do aluno de graduação, além dos percentuais estabelecidos anteriormente para pesquisa ou extensão, devem ser ainda retiradas das despesas das instituições as despesas com o pagamento de inativos e pensionistas e pagamento de exercícios anteriores, por não constituírem gastos que se dirigem aos cursos de graduação das instituições. (AMARAL; PINTO, 2010, p. 6263)

Partindo dessa metodologia para a obtenção do custo aluno e utilizando-se dos dados relativos ao financiamento das IES contidos nos microdados do Censo da Educação Superior, dos dados referentes à titulação docente e matrículas de graduação obtidos nas sinopses estatísticas do Censo da Educação Superior, dos dados referentes aos discentes matriculados na pós-graduação stricto sensu e conceitos dos programas de pós-graduação da Capes, todos entre os anos de 2012 a 2016, será calculado o custo médio do aluno das UEs, por estado da federação, nesses cinco anos.

Amaral e Pinto (2010, p. 64) ressaltam que é preciso considerar que há cursos ofertados pelas IES, como os de "pós-graduação lato sensu, cursos de extensão, residência médica [...]", que não são considerados nessa metodologia "pela inexistência de dados sistematizados" (Ibid., p. 64). Nessa perspectiva, ressalta-se que os dados do custo aluno das UEs, por estado da federação, não contemplam essas demais atividades oferecidas por essas instituições. E, caso esses dados fossem disponibilizados pelo Censo da Educação Superior ou pela Capes, de forma sistematizada, eles poderiam compor essa metodologia, modificando o seu resultado. A UEG, por exemplo, em 6 de novembro de 2017, possuía $3.037^{3}$ alunos de pós-graduação lato sensu ofertados gratuitamente e que não foram contabilizados nessa análise. Se tivessem sido

\footnotetext{
${ }^{3}$ Informação fornecida pela instituição
} 
incorporados ao estudo, reduziria o valor do custo aluno que foi calculado para essa instituição, neste estudo.

Outro elemento a ser considerado nos dados do custo aluno das UEs, apurado neste estudo, é a origem das informações referentes ao seu financiamento, pois esses dados "são fornecidos pelas próprias instituições e, portanto, não há a garantia de que eles correspondam aos valores reais. De qualquer forma, na falta de outras fontes que possua os dados financeiros de todas UEs do país, os microdados do Inep "nos fornecem uma primeira aproximação de um universo ainda pouco conhecido" (AMARAL; PINTO, 2010, p. 57).

Para a realização de um estudo como este, que contempla um universo de 38 UEs distribuídas em 22 estados da federação, não há outra fonte de consulta que congregue todas as informações financeiras necessárias dessas instituições em um mesmo banco de dados. Portanto, este estudo utiliza do total das despesas apresentadas ao Inep, que são classificadas como: pessoal, remuneração de professores ativos, remuneração de pessoal técnicoadministrativo/pedagógico, benefícios e encargos sociais, outras despesas de custeio, investimentos (despesas de capital), despesas com pesquisa e desenvolvimento e outras despesas. Cabe ressaltar que consta da orientação para preenchimento do Censo da Educação Superior pela IES que os valores referentes às despesas de remuneração docente sejam dos docentes ativos, ou seja, não devem incluir despesas com pensionistas e inativos.

Conforme essa metodologia de cálculo, o valor líquido dos recursos financeiros destinado ao ensino das IES é obtido

\footnotetext{
subtraindo-se das despesas totais um percentual que se dirige às atividades de pesquisa ou extensão, além das seguintes despesas: pagamento de inativos e pensionistas e pagamentos de exercícios anteriores.

$\mathrm{O}$ percentual que se dirige às atividades de pesquisa ou extensão é calculado utilizando-se parâmetros que se relacionam ao percentual de professores em tempo integral, ao quantitativo de mestres e doutores em relação ao total de professores, quantitativo de estudantes de pós-graduação stricto sensu em relação ao total de estudantes das instituições e conceito médio Capes dos programas de pós-graduação. (AMARAL; PINTO, 2010, p. 64)
}

Para este estudo, realizou-se o cálculo do custo médio do aluno das UEs, por estado da federação, de 2012 a 2016. Por exemplo, como o estado do Rio de Janeiro possui duas UEs, a Uerj e a Uenf, os dados apurados referentes a esse estado contemplam essas duas instituições juntas, pois o objetivo deste estudo é analisar esses dados por estado e não por UE. Para o detalhamento da metodologia, utilizou-se, como exemplo, a obtenção do custo médio do aluno das UEs do ano de 2012. 
Tendo em vista as etapas da metodologia para apurar o valor do custo médio do aluno, proposta por Amaral e Pinto (2010), o primeiro passo seria a obtenção do fator de tempo integral, ou seja, o FTI, que é obtido pela divisão do número de funções docentes em tempo integral pelo número total de funções docentes. O fator relacionado ao percentual de professores em tempo integral (FTI) das UEs, por estado da federação, referente ao ano de 2012, consta da tabela 01 e será utilizado nas demais etapas.

Tabela 1 - Fator de tempo integral (FTI) das UEs, por estados da federação - ano de 2012

\begin{tabular}{|c|c|c|c|c|}
\hline Estado & Total de docentes & $\begin{array}{c}\text { Total de docentes em } \\
\text { Tempo Integral* }\end{array}$ & $\%$ & FTI \\
\hline $\mathrm{AM}$ & 1.819 & 1.040 & 57,2 & 0,57 \\
\hline $\mathrm{RR}$ & 222 & 193 & 86,9 & 0,87 \\
\hline $\mathrm{PA}$ & 1.185 & 991 & 83,6 & 0,84 \\
\hline $\mathrm{AP}$ & 150 & 70 & 46,7 & 0,47 \\
\hline TO & 148 & 84 & 56,8 & 0,57 \\
\hline MA & 1.288 & 304 & 23,6 & 0,24 \\
\hline PI & 1.419 & 305 & 21,5 & 0,21 \\
\hline $\mathrm{CE}$ & 1.899 & 1.473 & 77,6 & 0,78 \\
\hline $\mathrm{RN}$ & 1.048 & 740 & 70,6 & 0,71 \\
\hline $\mathrm{PB}$ & 1.038 & 938 & 90,4 & 0,90 \\
\hline $\mathrm{PE}$ & 1.349 & 844 & 62,6 & 0,63 \\
\hline $\mathrm{AL}$ & 614 & 264 & 43,0 & 0,43 \\
\hline $\mathrm{BA}$ & 5.044 & 3.848 & 76,3 & 0,76 \\
\hline $\mathrm{MG}$ & 2.150 & 1.015 & 47,2 & 0,47 \\
\hline RJ & 3.221 & 2.196 & 68,2 & 0,68 \\
\hline $\mathrm{SP}$ & 11.936 & 10.581 & 88,6 & 0,89 \\
\hline PR & 7.283 & 5.411 & 74,3 & 0,74 \\
\hline $\mathrm{SC}$ & 1.194 & 723 & 60,6 & 0,61 \\
\hline $\mathrm{RS}$ & 199 & 199 & 100,0 & 1,00 \\
\hline MS & 713 & 305 & 42,8 & 0,43 \\
\hline MT & $1.107,00$ & 463 & 41,8 & 0,42 \\
\hline GO & $2.336,00$ & 634 & 27,1 & 0,27 \\
\hline
\end{tabular}

* Os dados das funções docentes em tempo integral são referentes às funções docentes em exercício, pois a sinopse estatística do Censo da Educação Superior não fornece essa informação referente aos docentes em exercício e afastados.

Fonte: Inep - Censo da Educação Superior - 2012. Sistematização e cálculo realizado por este estudo, conforme metodologia proposta por Amaral e Pinto (2010).

O segundo passo da metodologia leva em consideração que existe uma relação direta entre o percentual das despesas destinadas à pesquisa ou à extensão e o número de docentes com a titulação de mestrado ou doutorado. A tabela 02 apresenta esses dados e o percentual 
referente ao quantitativo de mestres e doutores, que deve ser deduzido das despesas totais para a obtenção do custo médio do aluno.

Tabela 2 - Percentual de dedução das atividades de pesquisa e extensão das UEs, por estados da federação, considerando a titulação dos docentes e o FTI - ano de 2012

\begin{tabular}{c|r|r|r|r|r}
\hline Estado & $\begin{array}{c}\text { N. } \\
\text { docentes* }\end{array}$ & $\begin{array}{c}\text { Total de docentes } \\
\text { mestres ou } \\
\text { doutores* }\end{array}$ & $\begin{array}{c}\text { \% de docentes } \\
\text { mestres ou } \\
\text { doutores }\end{array}$ & \multicolumn{1}{c|}{ FTI } & \multicolumn{2}{c}{$\begin{array}{c}\text { \% de dedução } \\
\text { PES EXT } \\
\text { (Docentes Me + Dr**) }\end{array}$} \\
\hline AM & 1.819 & 1.044 & 57,4 & 0,57 & 16,4 \\
\hline RR & 222 & 123 & 55,4 & 0,87 & 24,1 \\
\hline PA & 1.185 & 709 & 59,8 & 0,84 & 11,2 \\
\hline AP & 150 & 72 & 48,0 & 0,47 & 15,7 \\
\hline TO & 148 & 82 & 55,4 & 0,57 & 6,2 \\
\hline MA & 1.288 & 672 & 52,2 & 0,24 & 4,7 \\
\hline PI & 1.419 & 623 & 43,9 & 0,21 & 27,7 \\
\hline CE & 1.899 & 1.354 & 71,3 & 0,78 & 21,3 \\
\hline RN & 1.048 & 633 & 60,4 & 0,71 & 34,0 \\
\hline PB & 1.038 & 781 & 75,2 & 0,90 & 19,2 \\
\hline PE & 1.349 & 829 & 61,5 & 0,63 & 11,1 \\
\hline AL & 614 & 317 & 51,6 & 0,43 & 28,3 \\
\hline BA & 5.044 & 3.736 & 74,1 & 0,76 & 12,2 \\
\hline MG & 2.150 & 1.108 & 51,5 & 0,47 & 27,6 \\
\hline RJ & 3.221 & 2.608 & 81,0 & 0,68 & 44,0 \\
\hline SP & 11.936 & 11.847 & 99,3 & 0,89 & 32,6 \\
\hline PR & 7.283 & 6.388 & 87,7 & 0,74 & 26,4 \\
\hline SC & 1.194 & 1.042 & 87,3 & 0,61 & 47,5 \\
\hline RS & 199 & 189 & 95,0 & 1,00 & 15,4 \\
\hline MS & 713 & 515 & 72,2 & 0,43 & 5,4 \\
\hline MT & 1.107 & 738 & 66,7 & 0,42 & \\
\hline GO & 2.336 & 927 & 39,7 & 0,27 & \\
\hline
\end{tabular}

* Funções docentes em exercício e afastados.

** Ver item 2 da metodologia para o cálculo do custo aluno.

Fonte: Inep - Censo da Educação Superior - 2012. Sistematização e cálculo realizado por este estudo, conforme metodologia proposta por Amaral e Pinto (2010).

A terceira etapa consiste em apurar o percentual de dedução das atividades de pesquisa e extensão referente ao percentual de matrículas de pós-graduação stricto sensu em relação às matrículas totais e ao FTI. Nesse sentido, a tabela 03 apresenta os dados referentes ao total de matrículas de graduação e pós-graduação stricto sensu das UEs por estado da federação, o quantitativo de matrículas da pós-graduação stricto sensu, o FTI e o percentual a ser deduzido para pesquisa e extensão referente ao item 3 da metodologia para o cálculo do custo aluno. 
Tabela 3 - Percentual de dedução das atividades de pesquisa e extensão das UEs, por estados da federação, considerando a presença de estudantes de pós-graduação stricto sensu e o FTI - ano de 2012

\begin{tabular}{c|r|r|r|r|r}
\hline Estado & $\begin{array}{c}\text { Total de matrículas } \\
\text { draduação* e pós- } \\
\text { graduação stricto } \\
\text { sensu }\end{array}$ & $\begin{array}{c}\text { Matrículas de } \\
\text { pós-graduação } \\
\text { stricto sensu }\end{array}$ & $\begin{array}{c}\text { \% estudantes na } \\
\text { pós-graduação } \\
\text { stricto sensu }\end{array}$ & FTI & $\begin{array}{c}\text { \% de dedução } \\
\text { PES EXT } \\
\text { Estudantes de } \\
\text { pós**) }\end{array}$ \\
\hline AM & 23.340 & 184 & 0,8 & 0,57 & 0,5 \\
\hline RR & 5.177 & 15 & 0,3 & 0,87 & 0,3 \\
\hline PA & 10.254 & 165 & 1,6 & 0,84 & 1,3 \\
\hline AP & 2.358 & 0 & 0,0 & 0,47 & 0,0 \\
\hline TO & 33.162 & 0 & 0,0 & 0,57 & 0,0 \\
\hline MA & 25.550 & 138 & 0,5 & 0,24 & 0,1 \\
\hline PI & 14.974 & 29 & 0,2 & 0,21 & 0,0 \\
\hline CE & 38.798 & 1.390 & 3,6 & 0,78 & 2,8 \\
\hline RN & 10.884 & 186 & 1,7 & 0,71 & 1,2 \\
\hline PB & 18.769 & 397 & 2,1 & 0,90 & 1,9 \\
\hline PE & 17.899 & 379 & 2,1 & 0,63 & 1,3 \\
\hline AL & 8.641 & 0 & 0,0 & 0,43 & 0,0 \\
\hline BA & 59.868 & 1.787 & 3,0 & 0,76 & 2,3 \\
\hline MG & 15.306 & 354 & 2,3 & 0,47 & 1,1 \\
\hline RJ & 33.374 & 4.305 & 12,9 & 0,68 & 8,8 \\
\hline SP & 161.342 & 39.033 & 24,2 & 0,89 & 21,4 \\
\hline PR & 72.950 & 5.217 & 7,2 & 0,74 & 5,3 \\
\hline SC & 13.086 & 833 & 6,4 & 0,61 & 0,9 \\
\hline RS & 2.228 & 0 & 0,0 & 1,00 & 0,0 \\
\hline MS & 7.977 & 171 & 2,1 & 0,43 & 0,2 \\
\hline MT & 14.378 & 181 & 1,3 & 0,42 & 0,6 \\
\hline GO & 18.517 & 105 & 0,27 & \\
\hline
\end{tabular}

* Matrículas de graduação presencial e a distância.

** Ver item 3 da metodologia para o cálculo do custo aluno.

Fonte: Geocapes - 2012. Sistematização e cálculo realizado por este estudo, conforme metodologia proposta por Amaral e Pinto (2010).

A quarta etapa, por sua vez, consiste em levantar o percentual de dedução referente à pesquisa e extensão, tendo em vista a média do conceito Capes dos cursos de pós-graduação stricto sensu das UEs. Os dados da tabela 04 apresentam qual é a dedução a ser realizada, considerando esse indicador nas UEs, por estado da federação, no ano de 2012. 
Tabela 4 - Percentual de dedução das atividades de pesquisa e extensão das UEs, por estados da federação, considerando a média dos conceitos Capes e o FTI - ano de 2012

\begin{tabular}{c|r|r|r}
\hline Estado & Conceito médio Capes & FTI & $\begin{array}{c}\text { \% deduça PESQ/EXT } \\
\text { (Média Capes)* }\end{array}$ \\
\hline AM & 3,5 & 0,57 & 2,2 \\
\hline RR & 3 & 0,87 & 2,6 \\
\hline PA & 3,16 & 0,84 & 2,7 \\
\hline AP & 0 & 0,47 & 0,0 \\
\hline TO & 0 & 0,57 & 0,0 \\
\hline MA & 3,2 & 0,24 & 0,8 \\
\hline PI & 3 & 0,21 & 0,6 \\
\hline CE & 3,7 & 0,78 & 3,3 \\
\hline RN & 3 & 0,71 & 2,1 \\
\hline PB & 3,36 & 0,90 & 3,3 \\
\hline PE & 3,41 & 0,63 & 2,3 \\
\hline AL & 0 & 0,43 & 0,0 \\
\hline BA & 3,45 & 0,76 & 2,9 \\
\hline MG & 3,55 & 0,47 & 1,9 \\
\hline RJ & 4,36 & 0,68 & 3,7 \\
\hline SP & 5,1 & 0,89 & 5,9 \\
\hline PR & 3,89 & 0,74 & 3,4 \\
\hline SC & 3,67 & 0,61 & 2,5 \\
\hline RS & 0 & 1,00 & 0,0 \\
\hline MS & 3,2 & 0,43 & 1,4 \\
\hline MT & 3,5 & 0,42 & 1,6 \\
\hline GO & 3 & 0,27 & 0,8 \\
\hline
\end{tabular}

*Ver item 4 da metodologia para o cálculo do custo aluno.

Fonte: Avaliação trienal Capes. Sistematização e cálculo realizado por este estudo, conforme metodologia proposta por Amaral e Pinto (2010).

A tabela 05 apresenta o percentual total de dedução referente às atividades de pesquisa e extensão que será utilizado para se obter o custo médio do aluno das UEs, no ano de 2012. Esse total é a soma dos percentuais obtidos nas tabelas 02, 03 e 04 . É pertinente observar que as UEs que já possuem, em seu quadro docente, o predomínio de professores em regime de tempo integral e composto por mestres e doutores, maior número proporcional de estudantes de pós-graduação em relação ao total de estudantes e maior média de conceito Capes, possuem também maiores percentuais de dedução relacionados à pesquisa e à extensão, segundo essa metodologia. Por exemplo, no estado de São Paulo, a dedução total será de 71,4\%, no Rio de Janeiro será de 40,1\% e no Paraná de 41,3\%. 
Tabela 5 - Percentual total de dedução referente às atividades de pesquisa e extensão para a obtenção do custo médio do aluno das UEs, por estado da federação - ano de 2012

\begin{tabular}{|c|c|c|c|c|}
\hline Estado & $\begin{array}{l}\text { \% PES EXT } \\
(\mathrm{Ms}+\mathrm{Dr})\end{array}$ & $\begin{array}{l}\text { \% PES EXT } \\
\text { (Est. Pós) }\end{array}$ & $\begin{array}{l}\text { \% PES EXT } \\
\text { (Média Capes) }\end{array}$ & $\begin{array}{l}\text { \% total de dedução referente à } \\
\text { PES/EXT do total das despesas }\end{array}$ \\
\hline $\mathrm{AM}$ & 16,4 & 0,5 & 2,2 & 19,1 \\
\hline $\mathrm{RR}$ & 24,1 & 0,3 & 2,6 & 26,9 \\
\hline $\mathrm{PA}$ & 25,0 & 1,3 & 2,7 & 29,1 \\
\hline AP & 11,2 & 0,0 & 0,0 & 11,2 \\
\hline $\mathrm{TO}$ & 15,7 & 0,0 & 0,0 & 15,7 \\
\hline MA & 6,2 & 0,1 & 0,8 & 7,1 \\
\hline PI & 4,7 & 0,0 & 0,6 & 5,4 \\
\hline $\mathrm{CE}$ & 27,7 & 2,8 & 3,3 & 33,7 \\
\hline $\mathrm{RN}$ & 21,3 & 1,2 & 2,1 & 24,6 \\
\hline PB & 34,0 & 1,9 & 3,3 & 39,2 \\
\hline $\mathrm{PE}$ & 19,2 & 1,3 & 2,3 & 22,9 \\
\hline $\mathrm{AL}$ & 11,1 & 0,0 & 0,0 & 11,1 \\
\hline $\mathrm{BA}$ & 28,3 & 2,3 & 2,9 & 33,4 \\
\hline MG & 12,2 & 1,1 & 1,9 & 15,1 \\
\hline RJ & 27,6 & 8,8 & 3,7 & 40,1 \\
\hline SP & 44,0 & 21,4 & 5,9 & 71,4 \\
\hline PR & 32,6 & 5,3 & 3,4 & 41,3 \\
\hline $\mathrm{SC}$ & 26,4 & 3,9 & 2,5 & 32,8 \\
\hline RS & 47,5 & 0,0 & 0,0 & 47,5 \\
\hline MS & 15,4 & 0,9 & 1,4 & 17,8 \\
\hline MT & 13,9 & 0,5 & 1,6 & 16,1 \\
\hline GO & 5,4 & 0,2 & 0,8 & 6,4 \\
\hline
\end{tabular}

Fonte: Dados das tabelas 58, 59 e 60. Sistematização e cálculo realizado por este estudo, conforme metodologia proposta por Amaral e Pinto (2010).

O gráfico 01 ilustra, pois, a variação do percentual total de dedução referente às atividades de pesquisa e extensão para o cálculo do custo aluno das UEs, por estado da federação, no ano de 2012. A leitura deste gráfico evidencia e ratifica a heterogeneidade desse conjunto de instituições que, embora possua a mesma categoria administrativa, ser universidade pública estadual, possui características institucionais distintas. Nesse sentido, os estados que apresentaram os menores percentuais de dedução foram Piauí (5,4\%), Goiás (6,4\%) e Maranhão $(7,1 \%)$ 
Gráfico 1 - Percentual total de dedução referente às atividades de pesquisa e extensão para a obtenção do custo médio do aluno das UEs, por estado da federação - ano de 2012

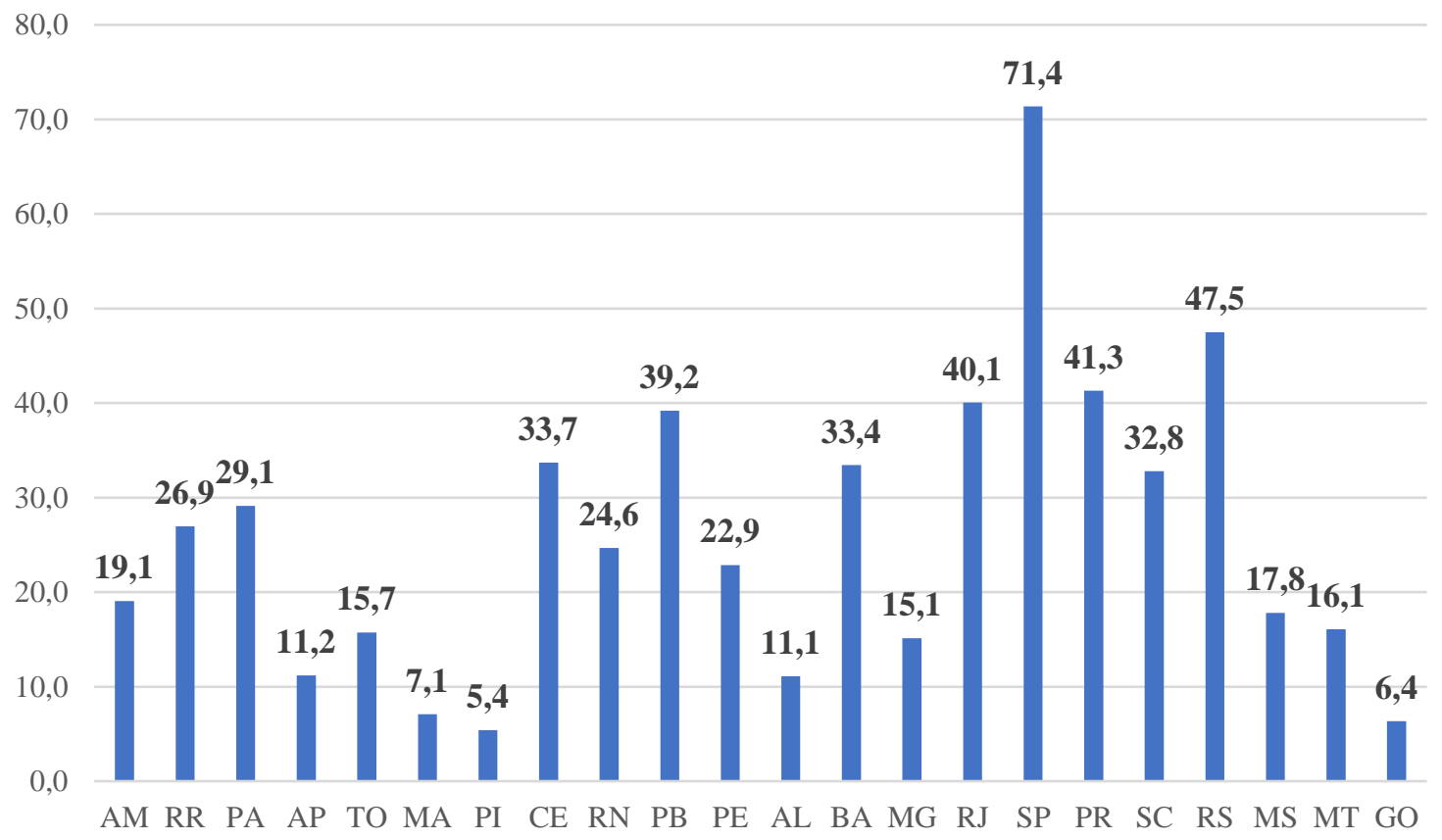

Fonte: Elaborado pela autora com base nos dados da tabela 5.

Por fim, a tabela 06 apresenta o total das despesas das UEs, por estado da federação, e o total das despesas efetivadas com o ensino e o custo médio do aluno no ano de 2012. Nesse ano, o custo médio do aluno das UEs no Brasil foi de $\mathrm{R} \$ 16.092,6$. Os dados do custo médio do aluno de 2012 serão utilizados juntamente com os demais dados obtidos e sistematizados por este estudo sobre o custo médio dos alunos das UEs de 2013, 2014, 2015 e 2016, para a obtenção da média do custo do aluno das UEs entre 2012 e $2016^{4}$.

Tabela 6 - Total das despesas efetivadas com o ensino de graduação e o custo médio do aluno das UEs, por estado da federação - ano de 2012

(Valores em R\$ a preços de janeiro de 2017 corrigidos pelo IPCA)

\begin{tabular}{c|r|r|r|r|r}
\hline Estado & $\begin{array}{c}\text { Total das despesas } \\
\text { das UEs em 2012 } \\
\text { (R\$) }\end{array}$ & $\begin{array}{c}\text { \% de } \\
\text { dedução } \\
\text { referente à } \\
\text { PES/EXT }\end{array}$ & $\begin{array}{c}\text { Total das despesas } \\
\text { efetivadas com } \\
\text { ensino }\end{array}$ & $\begin{array}{c}\text { Total de alunos de } \\
\text { graduação e pós- } \\
\text { graduação stricto sensu }\end{array}$ & $\begin{array}{c}\text { Custo médio } \\
\text { do aluno }\end{array}$ \\
\hline AM & 430.862 .022 & 19,1 & 348.681 .191 & 23.340 & $14.939,2$ \\
\hline RR & 248.541 .018 & 26,9 & 181.574 .416 & 5.177 & $35.073,3$ \\
\hline PA & 237.985 .650 & 29,1 & 168.715 .765 & 10.254 & $16.453,7$ \\
\hline AP & 15.424 .549 & 11,2 & 13.696 .999 & 33.162 & $5.808,7$ \\
\hline TO & 30.179 .345 & 15,7 & 25.434 .199 & 25.550 & $18.201,8$ \\
\hline MA & 500.464 .255 & 7,1 & 465.054 .736 & & 767,0 \\
\hline
\end{tabular}

${ }^{4}$ Os dados referentes ao custo médio dos alunos das UEs, por estado da federação, referente ao período de 2013 a 2016, foram obtidos por cálculos realizados por este estudo e serão apresentados já sistematizados. 
Conclusão

\begin{tabular}{|c|c|c|c|c|c|}
\hline PI & 222.820 .675 & 5,4 & 210.777 .598 & 14.974 & $14.076,2$ \\
\hline $\mathrm{CE}$ & 458.538 .594 & 33,7 & 303.969 .228 & 38.798 & $7.834,7$ \\
\hline $\mathrm{RN}$ & 176.800 .142 & 24,6 & 133.219 .428 & 10.884 & $12.239,9$ \\
\hline $\mathrm{PB}$ & 358.297 .306 & 39,2 & 217.888 .442 & 18.769 & $11.609,0$ \\
\hline PE & 571.181 .357 & 22,9 & 440.525 .962 & 17.899 & $24.611,8$ \\
\hline $\mathrm{AL}$ & 80.866 .572 & 11,1 & 71.890 .931 & 8.641 & $8.319,7$ \\
\hline BA & 1.354 .035 .413 & 33,4 & 901.525 .013 & 59.868 & $15.058,5$ \\
\hline $\mathrm{MG}$ & 398.777 .148 & 15,1 & 338.453 .477 & 15.306 & $22.112,5$ \\
\hline $\mathrm{RJ}$ & 1.570.398.277 & 40,1 & 941.240 .310 & 33.374 & $28.202,8$ \\
\hline $\mathrm{SP}$ & 11.079 .921 .062 & 71,4 & 3.173 .628 .782 & 161.342 & $19.670,2$ \\
\hline PR & 1.902 .225 .800 & 41,3 & 1.116 .941 .494 & 72.950 & $15.311,1$ \\
\hline $\mathrm{SC}$ & 361.487 .299 & 32,8 & 242.907 .891 & 13.086 & $18.562,4$ \\
\hline $\mathrm{RS}$ & 53.266 .985 & 47,5 & 27.971 .859 & 2.228 & $12.554,7$ \\
\hline MS & 117.134 .352 & 17,8 & 96.285 .679 & 7.977 & $12.070,4$ \\
\hline MT & 188.299 .457 & 16,1 & 158.004 .313 & 14.378 & $10.989,3$ \\
\hline $\mathrm{GO}$ & 234.157 .463 & 6,4 & 219.280.958 & 18.517 & $11.842,1$ \\
\hline Brasil & 20.591.664.741 & & 9.797.668.671 & 608.832 & $16.092,6$ \\
\hline
\end{tabular}

Fonte: Inep - Censo da Educação Superior e Geocapes - 2012. Sistematização e cálculo realizado por este estudo, conforme metodologia proposta por Amaral e Pinto (2010).

A tabela 07 apresenta os dados referentes ao custo médio do aluno das UEs, por estado da federação, no período de 2012 a 2016, já corrigidos pelo IPCA a preços de janeiro de 2017. Nesse período, o custo médio do aluno de graduação nas UEs do Brasil foi de $\mathrm{R} \$ 15.281,93$. De acordo com a metodologia utilizada, os dados institucionais (financeiros, matrículas e funções docentes) coletados no Censo da Educação Superior e os dados da Capes acerca da pósgraduação stricto sensu, os estados do Tocantins (R \$3.732,23), Amapá (R\$6.518,31), Ceará (R \$7.316,14), Piauí (R \$8.579,11) e Paraíba $(\mathrm{R} \$ 9.052,64)$ apresentaram os mais baixos valores de média do custo aluno de suas UEs, entre os anos de 2012 e 2016.

Tabela 07 - Custo médio do aluno das universidades estaduais brasileiras, por estado da federação - 2012 a 2016 (Valores em $\mathrm{R} \$$ a preços de janeiro de 2017 corrigidos pelo IPCA)

Continua

\begin{tabular}{c|r|r|r|r|r|r}
\hline Estado & \multicolumn{1}{c|}{$\mathbf{2 0 1 2}$} & \multicolumn{1}{c|}{$\mathbf{2 0 1 3}$} & \multicolumn{1}{c|}{$\mathbf{2 0 1 4}$} & \multicolumn{1}{c|}{$\mathbf{2 0 1 5}$} & \multicolumn{1}{c}{$\mathbf{2 0 1 6}$} & \multicolumn{1}{c}{ Média } \\
\hline AM & $14.939,2$ & $10.440,4$ & $14.354,9$ & $11.497,8$ & $10.292,5$ & $\mathbf{1 2 . 3 0 4 , 9 4}$ \\
\hline RR & $35.073,3 * *$ & $8.814,2$ & $8.703,4$ & $11.640,2$ & $10.190,8$ & $\mathbf{1 4 . 8 8 4 , 3 5}$ \\
\hline PA & $16.453,7$ & $14.319,3$ & $14.918,8$ & $16.796,1$ & $13.499,5$ & $\mathbf{1 5 . 1 9 7 , 4 8}$ \\
\hline AP & $5.808,7$ & $7.566,3$ & $5.743,1$ & $5.271,1$ & $8.202,3$ & $\mathbf{6 . 5 1 8 , 3 1}$ \\
\hline TO & 767,0 & $2.929,9$ & $4.624,7$ & $4.848,9$ & $5.490,7$ & $\mathbf{3 . 7 3 2 , 2 3}$ \\
\hline MA & $18.201,8$ & $12.117,6$ & $10.830,3$ & $12.479,5$ & $13.887,1$ & $\mathbf{1 3 . 5 0 3 , 2 7}$ \\
\hline PI & $14.076,2$ & $9.149,7$ & $9.322,2$ & $3.832,2$ & $6.515,2$ & $\mathbf{8 . 5 7 9 , 1 1}$ \\
\hline CE & $7.834,7$ & $8.287,4$ & $7.325,2$ & $6.283,3$ & $6.850,1$ & $\mathbf{7 . 3 1 6 , 1 4}$ \\
\hline RN & $12.239,9$ & $16.821,2$ & $16.266,6$ & $18.832,5$ & $12.945,2$ & $\mathbf{1 5 . 4 2 1 , 1 0}$ \\
\hline
\end{tabular}


Conclusão

\begin{tabular}{c|r|r|r|r|r|r}
\hline PB & $11.609,0$ & $3.098,7$ & $9.677,8$ & $11.719,9$ & $9.157,8$ & $\mathbf{9 . 0 5 2 , 6 4}$ \\
\hline PE & $24.611,8$ & $20.848,2$ & $22.008,8$ & $21.526,1$ & $20.638,3$ & $\mathbf{2 1 . 9 2 6 , 6 3}$ \\
\hline AL & $8.319,7 *$ & $21.737,7$ & $11.703,8$ & $10.979,7$ & $10.068,4$ & $\mathbf{1 2 . 5 6 1 , 8 8}$ \\
\hline BA & $15.058,5$ & $14.593,9$ & $14.743,5$ & $17.076,9$ & $12.791,5$ & $\mathbf{1 4 . 8 5 2 , 8 8}$ \\
\hline MG & $22.112,5$ & $25.118,3$ & $18.419,4$ & $13.556,2$ & $13.888,2$ & $\mathbf{1 8 . 6 1 8 , 9 1}$ \\
\hline RJ & $28.202,8$ & $31.559,0$ & $28.848,8$ & $12.109,0$ & $15.539,8$ & $\mathbf{2 3 . 2 5 1 , 8 8}$ \\
\hline SP & $19.670,2$ & $21.791,3$ & $16.029,9$ & $18.780,4$ & $14.172,7$ & $\mathbf{1 8 . 0 8 8 , 9 1}$ \\
\hline PR & $15.311,1$ & $17.279,7$ & $16.630,2$ & $14.021,4$ & $18.677,8$ & $\mathbf{1 6 . 3 8 4 , 0 2}$ \\
\hline SC & $18.562,4$ & $17.199,2$ & $18.093,0$ & $16.308,5$ & $16.816,1$ & $\mathbf{1 7 . 3 9 5 , 8 6}$ \\
\hline RS & $12.554,7$ & $13.637,0$ & $15.678,0$ & $13.471,2$ & $11.968,0$ & $\mathbf{1 3 . 4 6 1 , 7 8}$ \\
\hline MS & $12.070,4$ & $12.985,2$ & $15.783,9$ & $20.961,1$ & $10.331,5$ & $\mathbf{1 4 . 4 2 6 , 4 1}$ \\
\hline MT & $10.989,3$ & $13.789,1$ & $15.861,3$ & $15.847,8$ & $12.151,3$ & $\mathbf{1 3 . 7 2 7 , 7 7}$ \\
\hline GO & $11.842,1$ & $14.738,3$ & $15.880,1$ & $14.795,8$ & $14.547,3$ & $\mathbf{1 4 . 3 6 0 , 7 2}$ \\
\hline Brasil & $\mathbf{1 6 . 0 9 2 , 6}$ & $\mathbf{1 6 . 8 8 4 , 5}$ & $\mathbf{1 5 . 3 6 1 , 8}$ & $\mathbf{1 4 . 5 8 5 , 3}$ & $\mathbf{1 3 . 4 8 5 , 4}$ & $\mathbf{1 5 . 2 8 1 , 9 3}$ \\
\hline
\end{tabular}

Fonte: Dados da tabela 63 para o ano de 2012 e para os demais anos, cujos cálculos foram realizados por meio dos dados contidos no apêndice VII. Sistematização e cálculo realizado por este estudo, conforme metodologia proposta por Amaral e Pinto (2010).

Os estados de Amapá, Tocantins, Piauí e Ceará foram os que apresentaram os menores valores de custo médio aluno de suas UEs, no período de 2012 a 2016. No Amapá, o custo aluno variou entre $\mathrm{R} \$ 5.271,1$ e R $\$ 8.202,3$. No estado do Tocantins, a Unitins, como já relatado anteriormente, vivenciou um processo de descredenciamento de seus cursos, acarretando grande oscilação em suas matrículas. Assim, o custo do aluno nessa instituição variou entre R \$767,0 e R\$5.490,7. No estado do Piauí, a oscilação no custo aluno da Uespi foi de $\mathrm{R} \$ 14.076,24$ a $\mathrm{R} \$ 3.832,2$ e no estado do Ceará, que possui três UEs, a Uece, a Urca e a UVA, a variação no custo médio do aluno dessas instituições foi de $\mathrm{R} \$ 6.283,3$ a $\mathrm{R} \$ 8.287,4$. Grande parte dos estados apresentou redução no valor do custo médio do aluno de suas UEs, no período entre 2012 e 2016. O custo médio dos alunos nas UEs, por estado da federação, está ilustrado nos dados do gráfico 02 . 
Gráfico 2 - Média do custo aluno das UEs, por estado da federação - 2012 a 2016

(Valores em R \$ a preços de janeiro de 2017 corrigidos pelo IPCA)

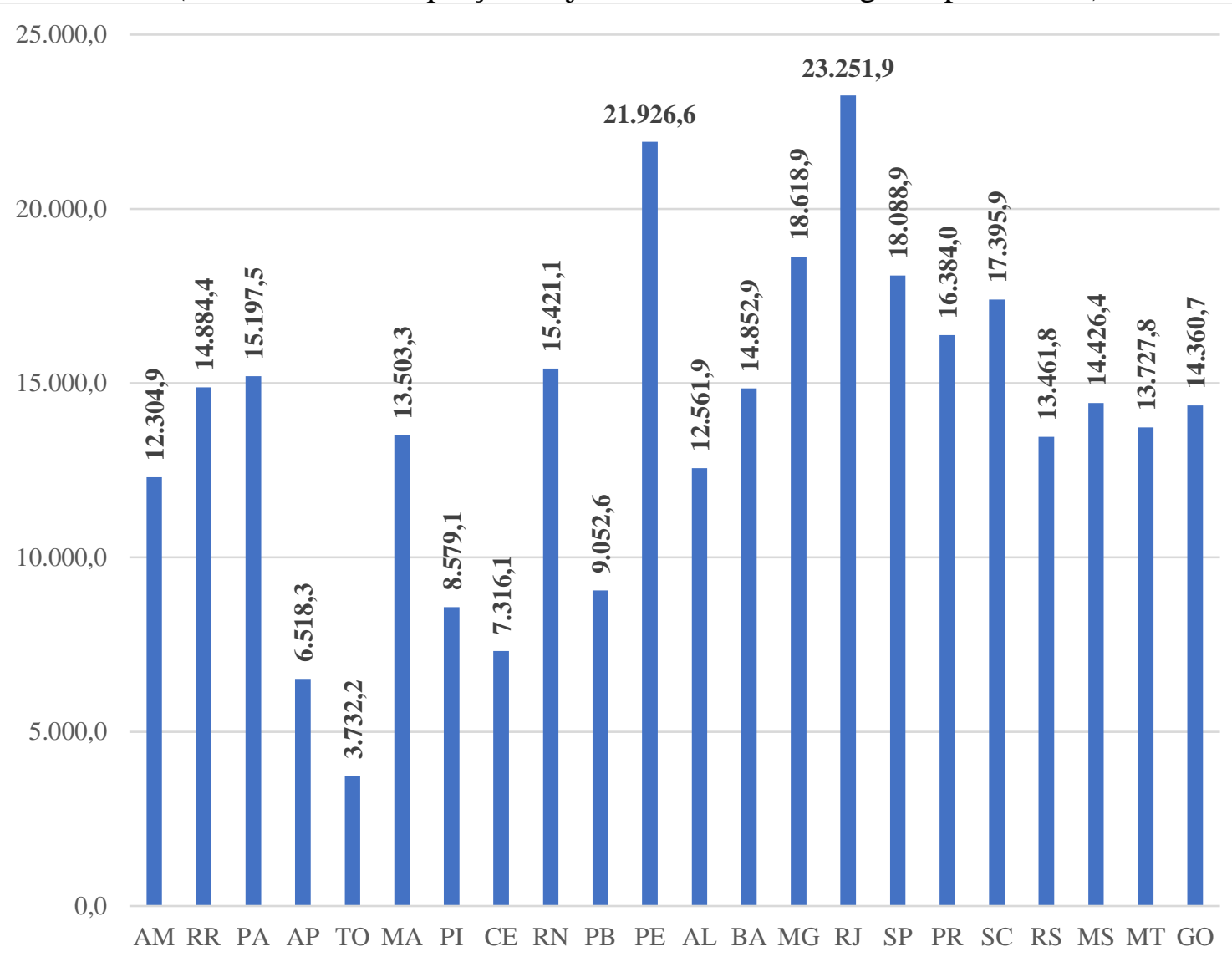

Fonte: Elaborado pela autora com base nos dados da tabela 7.

Dentre as UEs do país, os estados que apresentaram os maiores valores referentes à média do custo aluno foram os seguintes estados: Rio de Janeiro ( $\mathrm{R}$ 23.251,9), Pernambuco (R $\$ 21.926,6)$, São Paulo (R $\$ 18.088,9)$, Paraná (R\$16.384,0) e Santa Catarina $(\mathrm{R} \$ 17.395,9)$. Dentre os estados da região Norte, Nordeste e Centro-Oeste, somente Rio Grande do Norte e Pernambuco apresentaram média do custo aluno de suas UEs superiores à média do país; e, dentre os estados da região Sudeste e Sul, apenas o Rio Grande do Sul obteve média do custo aluno da Uergs inferior à média do custo das UEs, no Brasil. O gráfico 03 ilustra a variação da média do custo aluno das UEs, no Brasil. 
Gráfico 3 - Evolução da média do custo aluno das UEs no Brasil - 2012 a 2016

(Valores em $\mathrm{R} \$$ a preços de janeiro de 2017 corrigidos pelo IPCA)

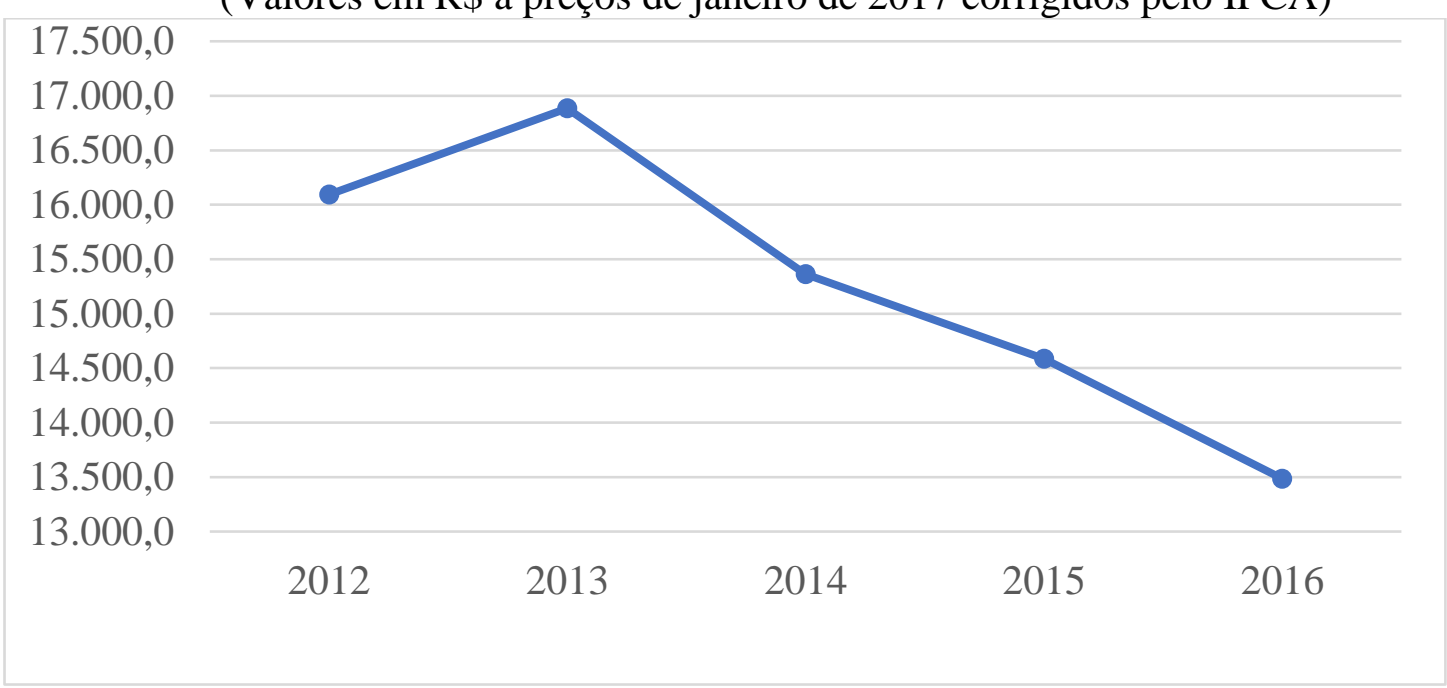

Fonte: Elaborado pela autora com base nos dados da tabela 7.

Considerando a média do custo aluno das UEs no Brasil, entre 2012 e 2016, os dados mostram que há uma redução nesse valor no país desde 2013. No ano de 2012, esse custo foi de $\mathrm{R} \$ 16.092,6$, chegando a $\mathrm{R} \$ 13.485,4$, em 2016. A análise do custo aluno das UEs, por estado da federação, mostrou que também existem consideráveis assimetrias entre as UEs, quando elas são observadas pelo prisma do financiamento. Ficou evidente ainda a desigualdade entre as UEs, por regiões da federação, visto que os mais baixos valores do custo médio aluno foram identificados nos estados das regiões Norte, Nordeste (com exceção da UPE) e Centro-Oeste, e os mais elevados, nas regiões Sudeste e Sul.

Amaral e Pinto (2010), utilizando o mesmo banco de dados adotado por esta pesquisa, também apuraram o valor do custo aluno das IES brasileiras, por categoria administrativa, referente ao ano de 2005, já corrigido pelo IPCA a preços de janeiro de 2010. Segundo esses autores, o custo do aluno das IES federais foi de $\mathrm{R} \$ 12.231,78$, das estaduais de $\mathrm{R} \$ 10.228,60$, das municipais de $\mathrm{R} \$ 9.480,31$, das particulares de $\mathrm{R} \$ 7.922,08$ e das comunitárias/confessionais e filantrópicas de $\mathrm{R} \$ 9.241,18$. Esses valores corrigidos pelo IPCA a preços de janeiro de 2017 correspondem a $\mathrm{R} \$ 18.827,18$ (federais), $\mathrm{R} \$ 15.743,88$ (estaduais), $\mathrm{R} \$ 14.592,11$ (municipais), $\mathrm{R} \$ 12.193,68$ (particulares) e R \$14.224,04 (comunitárias/confessionais e filantrópicas).

\section{Considerações finais}

A metodologia adotada por este estudo para apurar o custo aluno das UEs permite tecer considerações mais consistentes e críticas em contraposição a análises simplistas que se ocupam apenas em levantar o gasto institucional por aluno, isto é, dividir o volume total das despesas 
da instituição pelo quantitativo de alunos matriculados. Esse tipo de análise que mostra somente o gasto por aluno pode acarretar em compreensões imprecisas e induzir a considerações falseadas que desconsideram o perfil acadêmico de cada IES e as suas diferenças institucionais. Por exemplo, se a simples divisão do volume das despesas pelo quantitativo de alunos da graduação for realizada desconsiderando as matrículas e atuação da IES na pós-graduação stricto-sensu, poderá gerar informação de um valor de gasto aluno muito alto que não corresponde à realidade da instituição. A incorporação das despesas realizadas com pagamento de inativos e as realizadas com pesquisa e extensão podem manipular esse dado e favorecer a compreensões equivocadas. Observa-se, então, que é necessário estabelecer critérios sólidos que favoreçam a realização de interpretações consistentes e não falaciosas.

A proposta de cálculo para obtenção do custo aluno apresentada por Amaral e Pinto (2010) requer um conjunto de dados acadêmicos e financeiros das instituições apreciadas. O tratamento desses dados referentes às UEs promovido por este estudo revelou um cenário de muita disparidade e assimetria no conjunto dessas IES. Por exemplo, para a obtenção do FTI foi necessário apurar o percentual de docentes das UEs que trabalham em regime de tempo integral. Essas informações mostraram que há uma grande diferença quanto a esse indicador nessas instituições, enquanto a UE do estado da Paraíba possuía 90,4\% de seus docentes em regime de tempo integral no ano de 2012, a Uespi, no estado do Piauí, possuía somente 21,5\%.

A assimetria existente no conjunto dessas instituições também pode ser observada nos dados apresentados nas tabelas 02 e 03 . Na primeira, foi possível identificar a notória assimetria quanto a formação do corpo docente das UEs no país, enquanto as estaduais paulistas possuíam 99,3\% de seu quadro docente composto por mestres e/ou doutores no ano de 2012, a UEG, no estado de Goiás possuía apenas 39,7\%. A apreciação dos dados da tabela 03 mostrou um indicador que possibilita refletir sobre a atuação das UEs na oferta de pós-graduação strictosensu. Nesse mesmo ano, enquanto as UEs paulistas possuíam 24,2\%, isto é, 39.033 estudantes cursando mestrado ou doutorado, as dos estados do Amapá, Tocantins, Alagoas e Rio Grande do Sul, não possuíam nenhuma matrícula nessa fase formativa.

Os dados referentes aos percentuais de dedução referente às atividades de pesquisa e extensão para a obtenção do custo médio do aluno do aluno das UEs, por estado da federação, ratificou as assimetrias institucionais e acadêmicas presentes nesse subcampo da educação superior no Brasil. Quanto menor for o percentual de dedução apresentado, mais concentrada é a atuação dessas instituições nas atividades vinculadas ao ensino em detrimento de baixa atuação na pesquisa e extensão. A assimetria quanto a esse indicador obtida por este estudo 
para as UEs foi muito considerável, pois variou de um percentual de dedução de $71,4 \%$ para as UEs paulistas a 5,4\% para a Uespi no estado do Piauí.

A análise do conjunto dos dados das UEs também revelou um fator importante que é característico no contexto social e educacional do país, as suas diferenças regionais. As UEs dos estados da região Norte, Nordeste e Centro-oeste são aos que possuem os mais baixos percentuais de seus docentes em regime de tempo integral, os mais baixos percentuais de seu quadro docente composto por mestres e doutores, as menores percentuais de seus alunos matriculados na pós-graduação stricto-sensu que, por sua vez, geraram os menores percentuais de dedução de despesas de pesquisa e extensão. É preciso evidenciar ainda que mesmo possuindo baixos percentuais de dedução para as atividades de pesquisa e extensão, grande parte dos estados dessas regiões apresentou valores de custo aluno de suas UEs bem inferiores aos apurados para as UEs das regiões Sul e Sudeste.

Este estudo identificou que ocorreu uma redução no valor do custo médio das UEs no Brasil no período analisado. No ano de 2012, ele foi de $\mathrm{R} \$ 16.092,60$ e em 2016, de $\mathrm{R} \$ 13.485,40$. O levantamento de informações realizadas para apurar o custo médio dos alunos das UEs brasileiras, por estado da federação, mostrou que não há homogeneidade entre os valores obtidos. Isso porque esses dados evidenciaram que, quanto a esse indicador, são perceptíveis as discrepâncias no tocante à destinação do volume de recursos, por aluno, promovidos pelos estados para fomentar as atividades de ensino promovidas por suas UEs. Portanto, são nítidas as diferenças regionais no custo médio do aluno das UEs, por estado da federação. Essa variação foi de um custo médio do aluno das UEs do estado do Rio de Janeiro de $\mathrm{R} \$ 23.251,90$ e o da Unitins de $\mathrm{R} \$ 3.732,20$.

Por tudo isso, conclui-se que as UEs apresentam características institucionais e acadêmicas muito heterogêneas que precisam ser desveladas. Este estudo mostrou que quanto ao custo aluno dessas instituições, levantado por meio de uma modelagem que utiliza uma ampla variedade de informações, existem preocupantes desigualdades nos valores obtidos. A correção dessas assimetrias e a ampliação no financiamento das UEs é condição essencial para que elas possam melhorar suas condições materiais e acadêmicas e elevar a qualidade, socialmente referenciada, de suas atividades indissociáveis de ensino, pesquisa e extensão para serem universidades de forma plena. 


\section{Referências}

AMARAL, Nelson Cardoso; PINTO, José Marcelino de Rezende. O financiamento das IES brasileiras em 2005: recursos públicos, privados e custo dos alunos. Série Estudos -

Periódico do Programa de Pós-Graduação em Educação da UCDB. Campo Grande - MS, n. 30, p. 51-70, jul./dez. 2010.

BRASIL. Constituição da República Federativa do Brasil: 1988. Diário Oficial da União, Brasília, DF, Senado Federal, 1988.

BRASIL. Lei n. ${ }^{\circ}$ 9.394, de 26 de dezembro de 1996. Estabelece as Diretrizes e Bases da Educação Nacional. Brasília, DF, 1996.

BRASIL. Instituto Nacional de Estudos e Pesquisas Educacionais Anísio Teixeira (Inep). Sinopse Estatística do Censo da Educação Superior (2012 a 2016). Brasília, DF: Inep. Disponível em: http://portal.inep.gov.br/web/guest/sinopses-estatisticas-da-educacao-superior. Acesso em 03 jul. 2018.

BRASIL. Instituto Nacional de Estudos e Pesquisas Educacionais Anísio Teixeira (Inep). Microdados do Censo da Educação Superior (2012 a 2016). Brasília, DF: Inep. Disponível em: http://portal.inep.gov.br/web/guest/sinopses-estatisticas-da-educacao-superior. Acesso em 03 jul. 2018.

CARVALHO, Renata Ramos da Silva. Universidade Estadual de Goiás: histórico, realidade e desafios. 2013. 202 f. Dissertação (Mestrado em Educação) - Faculdade de Educação, Universidade Federal de Goiás, Goiânia, 2013.

FIALHO, Nadia Hage. Educação superior no Brasil: Universidades Estaduais à deriva? 2011. Disponível em: http://www.Uneb.br/gestec/publicacoes-e-recursos-parapesquisa/publicacoes/. Acesso em: 8 set. 2016.

FIALHO, Nadia Hage Universidades estaduais no Brasil: pauta para a construção de um sistema nacional articulado de educação. Revista da FAEEBA - Educação e Contemporaneidade, Salvador, v. 21, n. 38, p. 81-93, jul./dez. 2012.

FIALHO, Nadia Hage. Universidade Multicampi. Brasília, DF: Autores Associados; Plano Editora, 2005.

SAMPAIO, Helena; BALBACHEVSKY, Elizabeth; PEÑALOZA, Verónica. Universidades estaduais no Brasil: características institucionais. São Paulo: NUPES/USP, 1998.

(Documento de Trabalho n. 4). 